\title{
Histone deacetylase inhibition of cardiac autophagy in rats on a high-fat diet with low-dose streptozotocin-induced type 2 diabetes mellitus
}

\author{
TING-I LEE ${ }^{1,2}$, KUAN-JEN BAI ${ }^{3,4}$, YAO-CHANG CHEN ${ }^{5}$, TING-WEI LEE ${ }^{2,6}$, CHENG-CHIH CHUNG $^{6,7}$, \\ WEN-CHIH TSAI ${ }^{8}$, SHIN-YI TSAO ${ }^{9}$ and YU-HSUN KAO ${ }^{6,10}$
}

\begin{abstract}
${ }^{1}$ Department of General Medicine, School of Medicine, College of Medicine, Taipei Medical University, Taipei 11031; ${ }^{2}$ Division of Endocrinology and Metabolism, Department of Internal Medicine, Wan Fang Hospital, Taipei 11696; ${ }^{3}$ School of Respiratory Therapy, College of Medicine, Taipei Medical University, Taipei 11031; ${ }^{4}$ Division of Pulmonary Medicine, Department of Internal Medicine, Wan Fang Hospital, Taipei Medical University, Taipei 11696; ${ }^{5}$ Department of Biomedical Engineering, National Defense Medical Center, Taipei 11490; ${ }^{6}$ Graduate Institute of Clinical Medicine, College of Medicine, Taipei Medical University, Taipei 11031; ${ }^{7}$ Division of Cardiovascular Medicine, Department of Internal Medicine, Wan Fang Hospital, Taipei Medical University, Taipei $11696 ;{ }^{8}$ Division of Cardiology, Tzu-Chi General Hospital, Institute of Medical Sciences, Tzu-Chi University, Hualien 97004; ${ }^{9}$ Division of Endocrinology and Metabolism, Department of Internal Medicine, Sijhih Cathay General Hospital, New Taipei City 22174; ${ }^{10}$ Department of Medical Education and Research, Wan Fang Hospital, Taipei Medical University, Taipei 11696, Taiwan, R.O.C.
\end{abstract}

Received January 21, 2017; Accepted August 3, 2017

DOI: $10.3892 / \mathrm{mmr} .2017 .7905$

\begin{abstract}
Autophagy serves a role in preserving cellular homeostasis. Diabetes mellitus (DM) impairs cardiac autophagy and is associated with an accumulation of cytotoxic proteins that may provoke apoptosis and damage cardiomyocytes. Histone deacetylase (HDAC) inhibitors attenuate cardiac fibrosis and inflammation, and improve cardiomyopathy resulting from DM. However, the effect of HDAC inhibition on autophagy in DM cardiomyopathy has not been investigated. The purpose of the present study was to evaluate whether HDAC inhibition modulates cardiac autophagy and to investigate the potential mechanisms in type $2 \mathrm{DM}$ (T2DM) hearts. Electrocardiography was used to evaluate cardiac function and western blotting was used to evaluate protein expression in autophagy, the serine/threonine protein kinase mTOR (mTOR) signaling pathway, poly adenosine
\end{abstract}

Correspondence to: Professor Yu-Hsun Kao, Graduate Institute of Clinical Medicine, College of Medicine, Taipei Medical University, 250 Wuxing Street, Taipei 11031, Taiwan, R.O.C.

E-mail: yuhsunkao@gmail.com

Dr Shin-Yi Tsao, Division of Endocrinology and Metabolism, Department of Internal Medicine, Sijhih Cathay General Hospital, Lane 59, 2 Jiancheng Road, Sijhih, New Taipei City 22174, Taiwan, R.O.C.

E-mail: metaendocr@yahoo.com.tw

Key words: histone deacetylase inhibitor, diabetes mellitus, autophagy, cardiomyocytes diphosphate ribose polymerase 1 (PARP1), insulin signaling, advanced glycosylation end product-specific receptor (RAGE), and proinflammatory cytokines in control rats and in rats treated with a high-fat diet (60\% fat) and low-dose streptozotocin $(35 \mathrm{mg} / \mathrm{kg})$ in order to induce T2DM, with or without an HDAC inhibitor (MPT0E014; $50 \mathrm{mg} / \mathrm{kg} / \mathrm{rat}$ daily for 7 days). Compared with the control rats, T2DM and T2DM rats treated with MPT0E014 exhibited elevated blood glucose levels and similar body weights. However, T2DM rats treated with MPT0E014 and control rats had a smaller left ventricular end-diastolic diameter compared with the T2DM rats. The control and T2DM rats treated with MPT0E014 had greater protein expression of cardiac phosphorylated (p)-5' adenosine monophosphate-activated protein kinase $\alpha 2$, light chain 3-II, Beclin-1, glucose transporter 4, p-protein kinase B, and insulin receptor substrate-1 (Ser 307) compared with T2DM rats. In addition, control and T2DM rats treated with MPT0E014 had decreased cardiac protein expression of cleaved PARP1, p-mTOR-S2448, p-P70S6K-Thr-389, RAGE, tumor necrosis factor- $\alpha$, and interleukin- 6 compared with T2DM rats. The present study demonstrated that MPT0E014 may improve cardiac function in T2DM rats by modulating myocardial autophagy, inflammation and insulin signaling.

\section{Introduction}

Type 2 diabetes mellitus (T2DM) accounts for $\sim 90 \%$ of all cases of diabetes mellitus (DM) and imparts a 1.5-2-fold increase in morbidity compared with the general population (1). Cardiovascular dysfunction is a major morbidity and cause of mortality in T2DM patients. However, the mechanisms underlying the high prevalence of cardiovascular dysfunction in 
patients with T2DM have not been completely elucidated $(2,3)$ and therapeutic outcomes remain unsatisfactory.

Autophagy is a highly conserved process that preserves cellular homeostasis between normal and pathophysiological conditions (4). Although autophagy is primarily involved in cell survival, continuous activation may lead to autophagic or apoptotic cell death (5). Cardiomyocyte autophagy is a crucial adaptive response of the myocardium to preserve the cellular energy balance, particularly during stress (6,7). T2DM patients exhibit increased cardiac autophagy and cleavage of caspase-3, which was investigated by analysis of the right atrial appendages from subjects receiving coronary artery bypass graft surgery (8). Impaired cardiac autophagy has been reported in metabolic syndrome and T2DM animals $(9,10)$. Since insulin is able to modulate myocardial autophagy via phosphatidylinositol 3-kinase/RAC- $\alpha$ serine/threonine protein kinase (Akt) signaling to inhibit the serine/threonine protein kinase mTOR (mTOR) signaling pathway, cardiac autophagy is impaired in insulin resistant T2DM (11). In addition, under conditions of elevated nutrients, mTOR is activated in hyperglycemia, thereby inhibiting autophagy. A decrease in autophagy results in an aggregation of cytotoxic proteins and defective organelles that may provoke apoptosis and damage cardiomyocytes (12). Therefore, modulation of autophagy may decrease DM cardiomyopathy.

Histone deacetylases (HDACs) serve an essential role in regulating cell proliferation, migration and death. Previously, HDACs and inhibitors have been identified to be therapeutic targets for type $1 \mathrm{DM}$ and T2DM $(13,14)$. A pan HDAC inhibitor MPT0E014 has been demonstrated to decrease cardiac fibrosis and profibrotic signaling protein expression in a heart failure animal model (15). In addition, MPT0E014 has been demonstrated to regulate cardiac metabolism through its effects on peroxisome proliferator-activated receptors (PPARs) and inflammatory cytokines to reduce the accumulation of fatty acids in DM hearts (16). However, it is not clear whether HDAC inhibition is able to regulate cardiac autophagy in T2DM cardiomyopathy. Additionally, advanced glycation end products (AGEs) are involved in the pathogenesis of vascular damage resulting from hyperglycemia (17). AGEs cause detrimental effects by directly altering the structure and function of AGE-modified macromolecules, or by binding with the advanced glycosylation end product-specific receptor (RAGE) (18). However, the effect of HDAC inhibition on the expression of RAGE in DM cardiomyopathy remains unknown.

Therefore, the purpose of the present study was to investigate the effect of the HDAC inhibitor MPT0E014 on the regulation of myocardial autophagy in rats with high-fat diet (HFD) and low-dose streptozotocin (STZ)-induced T2DM.

\section{Materials and methods}

Animals, blood sampling and tissue preparation. The present study was approved by the Institutional Animal Care and Use Committee of Taipei Medical University (approval no. LAC-2013-0085). Rats were housed under standard environmental conditions $\left(21 \pm 2^{\circ} \mathrm{C}\right.$; humidity $50-60 \%$; $12-\mathrm{h}$ light/dark cycle) and maintained on commercial rat food and tap water ad libitum. A total of 24 male 8-week old Wistar rats, weighing $260 \pm 4.0 \mathrm{~g}$ were purchased from BioLasco, Taiwan, Co., Ltd., (Taipei, Taiwan) were used in the present study. To induce T2DM, 16 rats were fed an HFD (60\% fat, 18\% protein and $21 \%$ carbohydrates; Research Diet Inc., New Brunswick, NJ, USA) ad libitum starting at 8 weeks old and received a low dose of STZ (35 mg/kg; Sigma-Aldrich, Merck KGaA, Darmstadt, Germany) intraperitoneally at 10 weeks old (19). T2DM was defined as high fasting plasma glucose $(\geq 15 \mathrm{mmol} / \mathrm{l})$ and was measured with a glucometer (Ascensia Elite; Bayer, Pittsburgh, PA, USA) (20,21). At 12 weeks of age, the rats were grouped into three groups of eight rats each, including control, HFD + low-dose STZ-induced T2DM and MPT0E014-treated HFD + low-dose STZ-induced T2DM groups. MPT0E014 [a pan-HDAC inhibitor (15); $50 \mathrm{mg} / \mathrm{kg}$ in $50 \%$ polyethylene glycol 400 and $0.25 \%$ carboxymethyl cellulose (22)], or a vehicle $(1 \mathrm{ml} / \mathrm{kg}$ of $50 \%$ polyethylene glycol 400 and $0.25 \%$ carboxymethyl cellulose) was given once daily for 7 days by oral gavage in the studied rats. The rats were sacrificed with an intraperitoneal injection of sodium pentobarbital $(100 \mathrm{mg} / \mathrm{kg})$ at 13 weeks of age. Body weight was measured prior to sacrifice. Fasting plasma blood urea nitrogen, creatinine, cholesterol, triglyceride and high-density lipoprotein-cholesterol (HDL-C) were obtained with a SPOTCHEM analyzer (Arkray, Inc., Kyoto, Japan) using SPOTCHEM II Inorganic Phosphorous reagent strips. Plasma free fatty acid was measured using a Free Fatty Acid Quantitation kit (Sigma-Aldrich; Merck KGaA) and plasma fasting insulin was measured with a Mercodia Ultrasensitive Rat Insulin ELISA (Mercodia AB, Uppsala, Sweden). Transverse tissue pieces from the left ventricle (LV) weighing $0.55-0.65 \mathrm{~g}$ were snap-frozen in liquid nitrogen and stored at $-80^{\circ} \mathrm{C}$ for protein isolation.

Echocardiographic measurements. At 10 and 13 weeks of age, transthoracic echocardiography was performed using a Vivid I ultrasound cardiovascular system (GE Healthcare, Chicago, IL, USA) was performed under isoflurane anesthesia (5\% for induction and 2\% for maintenance) in the control and HFD + STZ T2DM rats with or without treatment with MPT0E014. M-mode tracing of the LV was used to measure the following cardiac structures: The LV end-diastolic diameter (LVEDd), LV end-systolic diameter (LVESd), interventricular septal thickness in diastole (IVSd), end diastolic volume (EDV), end systolic volume (ESV), fractional shortening (FS) and the ejection fraction (EF) (15).

Western blot analysis. Tissues were homogenized and lysized in M-PER ${ }^{\mathrm{TM}}$ Mammalian Protein Extraction Reagent (Thermo Fisher Scientific, Inc., Waltham, MA, USA) and the protein concentration were determination by Bradford assay. Equal amounts of proteins $(40 \mu \mathrm{g})$ were resolved by SDS-PAGE on a $8-15 \%$ gel followed by electrophoretic transfer of proteins onto polyvinylidene difluoride membranes. Blots were blocked with $5 \%$ skimmed milk for $1 \mathrm{~h}$ at room temperature, then probed with antibodies against Light Chain (LC) 3-I (cat. no. APG8B; 1:1,000; Abgent, Inc., San Diego, CA, USA) and LC3-II (cat. no. APG8B; 1:1,000; Abgent, Inc.), Beclin-1 (cat. no. ADI-905-721; 1:1,000; Enzo Life Sciences, Inc., Farmingdale, NY, USA), poly ADP-ribose polymerase 1 (PARP1; cat. no. sc-1561; 1:1,000; Santa Cruz Biotechnology, Inc., Dallas, TX, USA), 5' adenosine monophosphate-activated 
Table I. Physical and biochemical characteristics of the control, T2DM and T2DM rats treated with MPT0E014 at 13 weeks.

\begin{tabular}{|c|c|c|c|}
\hline Physical characteristics & Control & T2DM & MPT0E104-treated T2DM \\
\hline $\mathrm{BW}, \mathrm{g}$ & $369.2 \pm 10.4$ & $370.7 \pm 17.1$ & $366.2 \pm 11.0$ \\
\hline $\mathrm{HW}, \mathrm{g}$ & $1.3 \pm 0.1$ & $1.6 \pm 0.1^{\mathrm{a}}$ & $1.3 \pm 0.1^{\mathrm{b}}$ \\
\hline $\mathrm{HW} / \mathrm{BW}$ ratio, g/kg & $3.5 \pm 0.1$ & $4.2 \pm 0.2^{\mathrm{a}}$ & $3.6 \pm 0.1^{\mathrm{b}}$ \\
\hline \multicolumn{4}{|l|}{ Biochemical characteristics } \\
\hline Fasting blood glucose, mmol/l & $5.9 \pm 0.3$ & $19.1 \pm 0.9^{\mathrm{a}}$ & $12.9 \pm 1.8^{\mathrm{a}, \mathrm{b}}$ \\
\hline BUN, mmol/1 & $5.5 \pm 0.4$ & $5.5 \pm 0.4$ & $5.1 \pm 0.2$ \\
\hline Creatinine, $\mathrm{mmol} / \mathrm{l}$ & $34.2 \pm 6.8$ & $42.7 \pm 2.7$ & $39.8 \pm 4.4$ \\
\hline Cholesterol, mmol/1 & $1.8 \pm 0.2$ & $1.9 \pm 0.1$ & $1.7 \pm 0.1$ \\
\hline Triglyceride, mmol/l & $0.7 \pm 0.1$ & $2.5 \pm 0.4^{\mathrm{a}}$ & $1.4 \pm 0.2^{\mathrm{b}}$ \\
\hline HDL-C, mmol/l & $0.5 \pm 0.1$ & $0.3 \pm 0.1^{\mathrm{a}}$ & $0.5 \pm 0.1^{\mathrm{b}}$ \\
\hline Free fatty acid $\mu \mathrm{mol} / 1$ & $26.3 \pm 3.3$ & $56.5 \pm 6.6^{\mathrm{a}}$ & $38.7 \pm 3.2^{\mathrm{b}}$ \\
\hline Fasting insulin, pmol/l & $48.8 \pm 10.5$ & $28.2 \pm 6.6$ & $29.2 \pm 2.7$ \\
\hline
\end{tabular}

Values are expressed as the mean \pm standard error of the mean. $\mathrm{n}=6 .{ }^{\mathrm{a}} \mathrm{P}<0.05$ vs. control, ${ }^{\mathrm{b}} \mathrm{P}<0.05$ vs. T2DM. T2DM, type 2 diabetes mellitus; BUN, blood urea nitrogen; HDL-C, high density lipoprotein-cholesterol; HW, heart weight; BW, body weight.

protein kinase (AMPK) $\alpha 2$ (cat. no. 07-363; 1:500; Upstate Biotechnology, Inc., Lake Placid, NY, USA), phosphorylated (p)-AMPK $\alpha 2$ Thr172 (cat. no. 07-681; 1:1,000; EMD Millipore, Billerica, MA, USA), anti-histone H3 (cat. no. ab1791; 1:1,000; Abcam, Cambridge, MA, USA), anti-acetyl-histone $\mathrm{H} 3$ at Lys9 (cat. no. 06-942; 1:5,000; EMD Millipore), mTOR (cat. no. 2972; 1:1,000; Cell Signaling Technology, Inc., Danvers, MA, USA), p-mTOR-Ser-2448 (cat. no. 2971; 1:1,000; Cell Signaling Technology, Inc.), P70S6 kinase (cat. no. 9202; 1:1,000; Cell Signaling Technology, Inc.) and p-P70S6K-Thr 389 (cat. no. 9205; 1:1,000; Cell Signaling Technology, Inc.), tumor necrosis factor (TNF)- $\alpha$ (cat. no. AB1837P; 1:500; EMD Millipore), interleukin (IL)-6 (cat. no. ab6672; 1:1,000; Thermo Fisher Scientific, Inc.), advanced glycosylation end product-specific receptor (RAGE; cat. no. PA1-84173; 1:3,000; Thermo Fisher Scientific, Inc.), glucose transporter (GLUT) 4 (cat.no. ab654; 1:2,000; Abcam), insulin substrate receptor(IRS; cat. no. 2382; 1:1,000; Cell Signaling Technology, Inc.), p-IRS-1 at Ser307 (cat. no. 2381; 1:1,000; Cell Signaling Technology, Inc.), Akt (cat. no. 4685; 1:1,000; Cell Signaling Technology, Inc.) and p-Akt (cat. no. 4060; 1:3,000; Cell Signaling Technology, Inc.) for overnight at $4^{\circ} \mathrm{C}$ and secondary antibodies conjugated with horseradish peroxidase (Leinco Technologies, Inc., Fenton, MO, USA) for $1 \mathrm{~h}$ at room temperature. Bound antibodies were detected with an enhanced chemiluminescence detection system (EMD Millipore) and analyzed with AlphaEaseFC software (version 6.0; ProteinSimple, San Jose, CA, USA). Targeted bands were normalized to cardiac GAPDH (Sigma-Aldrich; Merck $\mathrm{KGaA}$ ) to confirm equal protein loading.

Statistical analysis. All quantitative data are expressed as the mean \pm standard error of the mean. Statistically significant differences between different groups was determined using one-way analysis of variance with Tukey's test for multiple comparisons as appropriate using SigmaStat (version 3.5; Systat Software, Inc., San Jose, CA, USA). P<0.05 was considered to indicate a statistically significant difference.

\section{Results}

Effect of MPTOE014 on biochemistry and cardiac function. The body weights were similar among rats in the control, HFD + low-dose STZ-induced T2DM and T2DM rats treated with MPT0E014 groups (Table I). However, HFD + low-dose STZ-induced T2DM rats had larger absolute heart weights compared with control rats and T2DM rats treated with MPT0E014. In addition, the heart-to-body weight ratios were greater in the HFD + low-dose STZ-induced T2DM rats compared with the control rats and T2DM rats treated with MPT0E014 (Table I). Compared with the control rats, HFD + low-dose STZ-induced T2DM rats and MPT0E014-treated T2DM rats had higher levels of blood glucose, triglycerides and free fatty acids (Table I). However, the MPT0E014-treated T2DM rats had lower levels of blood glucose, triglycerides and free fatty acids compared with HFD + low-dose STZ-induced T2DM rats. The level of HDL-C was lower in the HFD + low-dose STZ-induced T2DM rats compared with the control and MPT0E014-treated T2DM rats (Table I). Total cholesterol, blood urea nitrogen, creatinine and insulin levels were not significantly different between the three groups $(\mathrm{P}>0.05)$.

Table II illustrates echocardiograms of control, HFD + STZ-induced T2DM and MPT0E014-treated T2DM rats. The HFD + STZ-induced T2DM rats had higher LVEDd, LVESd, EDV and ESV values, and lower EF and FS values compared with control and MPT0E014-treated T2DM rats.

Effects of MPTOE014 on cardiac autophagy and cell death. Compared with the control group, the acetyl histone $\mathrm{H} 3$ (Ac-H3K9)/histone 3 (H3) ratio was decreased in the HFD + STZ-induced T2DM hearts by 0.87 . Ac-H3K9/H3 ratio was increased in the T2DM rats treated with MPT0E104 compared with the T2DM group (Fig. 1A). In order to assess the role of autophagy in T2DM cardiomyopathy, cardiac LC3-II protein expression was measured and it was 
Table II. Echocardiogram of control, T2DM and T2DM rats treated with MPT0E104 at 13 weeks.

\begin{tabular}{|c|c|c|c|c|c|c|}
\hline Group & LVEDd (mm) & LVEDs (mm) & $\mathrm{EDV}(\mathrm{mm})$ & $\mathrm{ESV}(\mathrm{mm})$ & $\mathrm{EF}(\%)$ & $\mathrm{FS}(\%)$ \\
\hline Control & $7.1 \pm 0.1$ & $3.0 \pm 0.2$ & $0.8 \pm 0.1$ & $0.08 \pm 0.1$ & $90.8 \pm 1.3$ & $57.9 \pm 2.6$ \\
\hline T2DM & $8.0 \pm 0.2^{\mathrm{a}}$ & $4.1 \pm 0.2^{\mathrm{a}}$ & $1.1 \pm 0.1^{\mathrm{a}}$ & $0.2 \pm 0.1^{\mathrm{a}}$ & $80.9 \pm 1.2^{\mathrm{a}}$ & $44.8 \pm 1.3^{\mathrm{a}}$ \\
\hline MPT0E104-treated T2DM & $7.1 \pm 0.2^{\mathrm{b}}$ & $3.2 \pm 0.2^{\mathrm{b}}$ & $0.8 \pm 0.1^{\mathrm{b}}$ & $0.09 \pm 0.1^{b}$ & $88.9 \pm 0.9^{b}$ & $53.1 \pm 0.5^{b}$ \\
\hline
\end{tabular}

Values are expressed as the mean \pm standard error of the mean. $\mathrm{n}=6 .{ }^{\mathrm{a}} \mathrm{P}<0.05 \mathrm{vs}$. control, ${ }^{\mathrm{b}} \mathrm{P}<0.05 \mathrm{vs}$. T2DM. T2DM, type 2 diabetes mellitus; LVEDd, left ventricular end-diastolic dimension; LVEDs, left ventricular end-systolic dimension; EDV, end-diastolic volume; ESV, end-systolic volume; EF, ejection fraction; FS, fractional shortening.
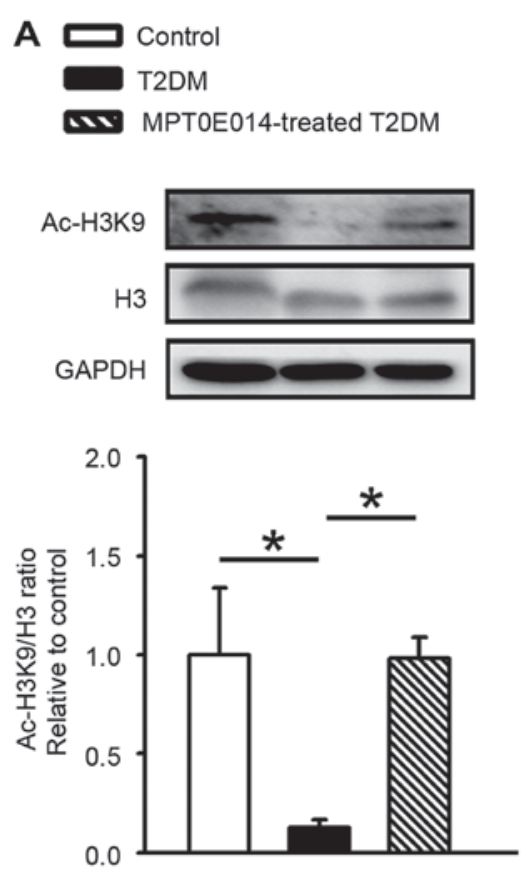

C
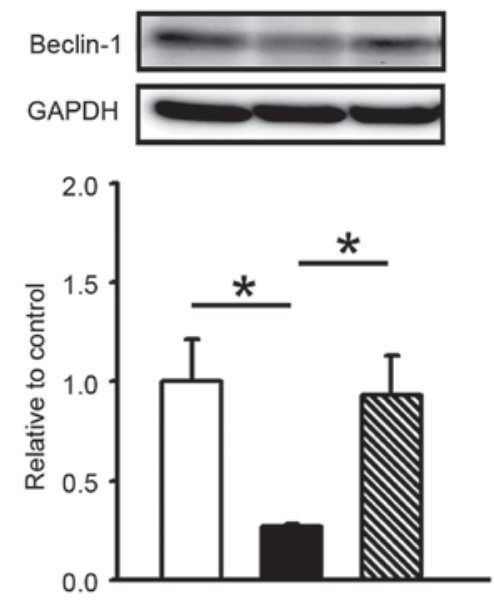

B
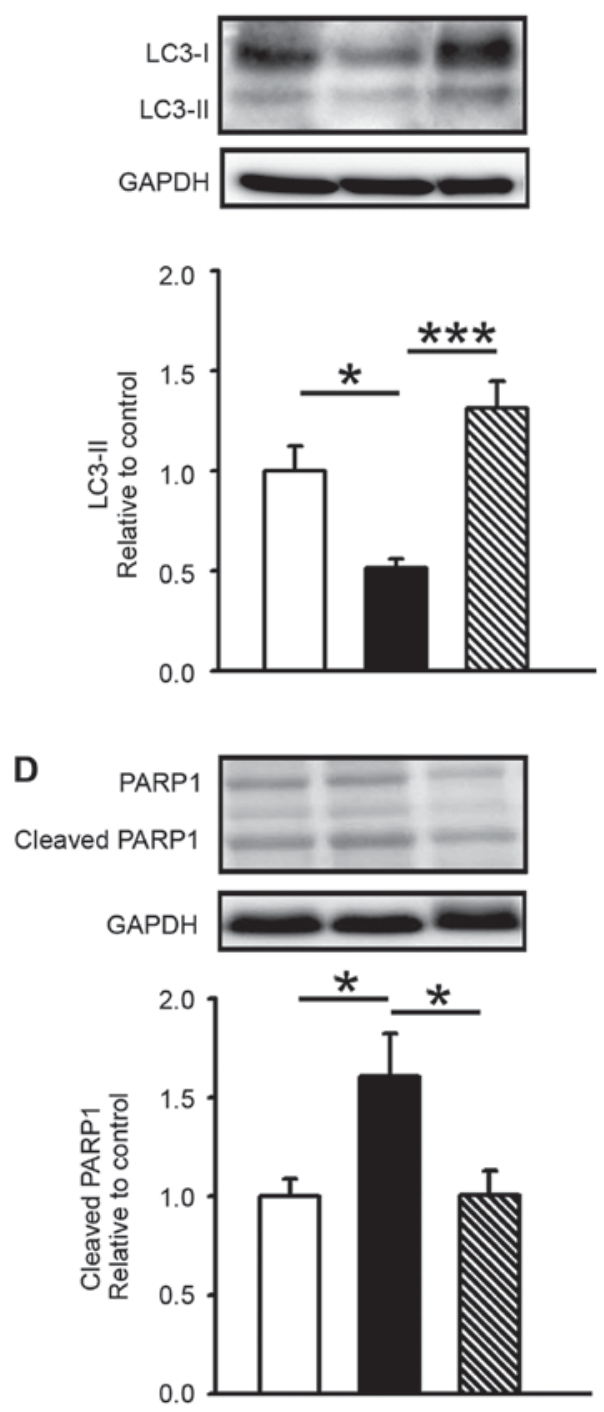

Figure 1. MPTOE014 increases myocardial autophagy and attenuates cell death in T2DM rats. T2DM rats induced with high-fat diet and low-dose streptozotocin had a decreased myocardial (A) AcH3K9/H3 ratio, and (B) LC3-II and (C) Beclin-1 level compared with the control and T2DM rats treated with MPT0E014. (D) T2DM rats had increased cardiac cleaved PARP1 protein compared with the control and T2DM rats treated with MPT0E014. Data were normalized to GAPDH and are presented as the mean \pm standard error of the mean. ${ }^{*} \mathrm{P}<0.05,{ }^{* * *} \mathrm{P}<0.001$. $\mathrm{n}=5$ rats/group. T2DM, type 2 diabetes mellitus; PARP1, poly ADP ribose polymerase; H3, histone 3; LC3-II, light chain 3; AcH3K9, acetyl-H3K9.

demonstrated that LC3-II levels significantly decreased in HFD + STZ-induced T2DM hearts compared with control hearts by $0.49(\mathrm{P}<0.05)$. Similar to control hearts, MPT0E014-treated T2DM hearts had higher LC3-II levels 


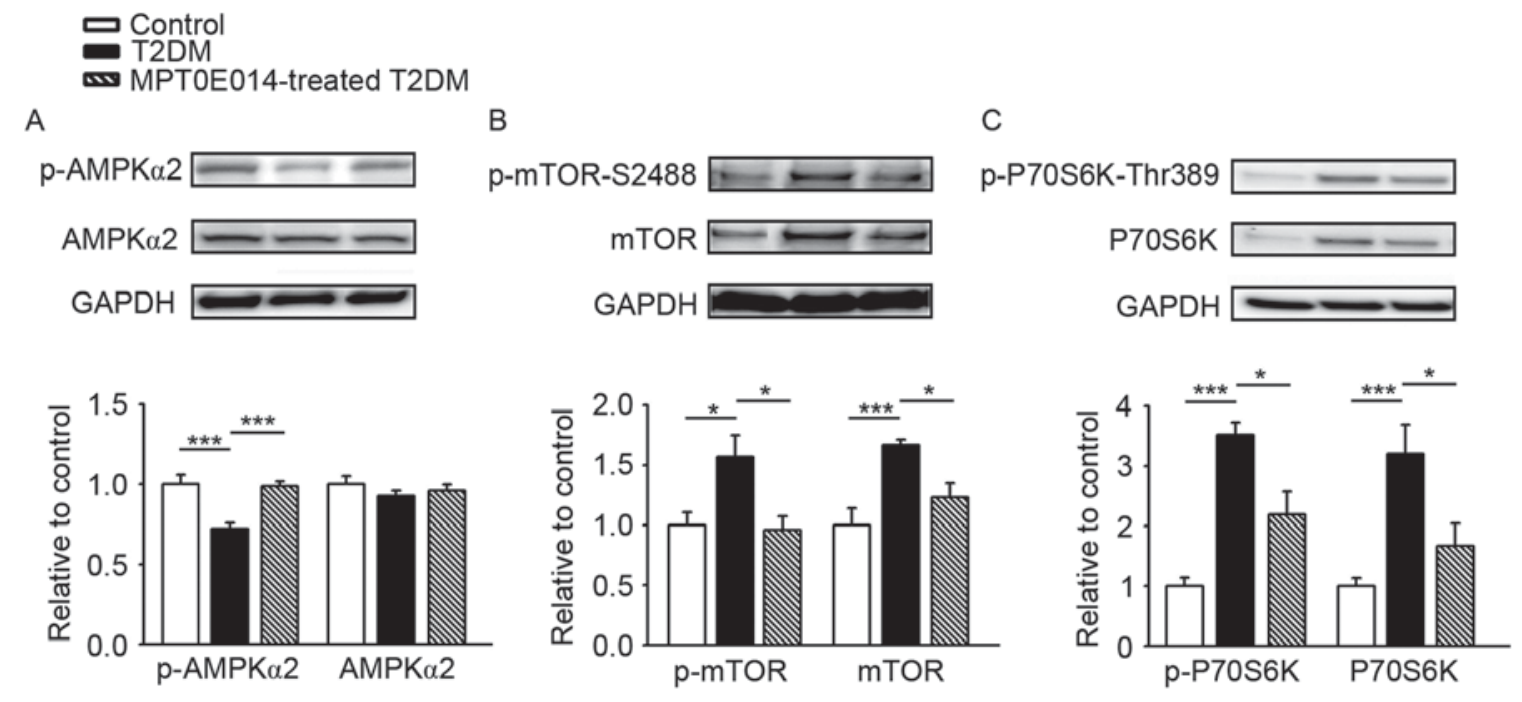

Figure 2. MPT0E014 reduces myocardial mTOR activity in T2DM rats. Western blots analysis of cardiac (A) total and p-AMPKa2, (B) total and p-mTOR-S2448, and (C) total and p-P70S6K at Thr389 levels in the control, high-fat diet low-dose streptozotocin induced T2DM rats and T2DM rats treated with MPT0E014. Data were normalized to GAPDH and are presented as the mean \pm standard error of the mean. ${ }^{*} \mathrm{P}<0.05,{ }^{* * * *} \mathrm{P}<0.001$. $\mathrm{n}=5$ rats/group. T2DM, type 2 diabetes mellitus; mTOR, serine/threonine protein kinase mTOR; p-, phosphorylated; AMPKa2, 5' adenosine monophosphate-activated protein kinase.
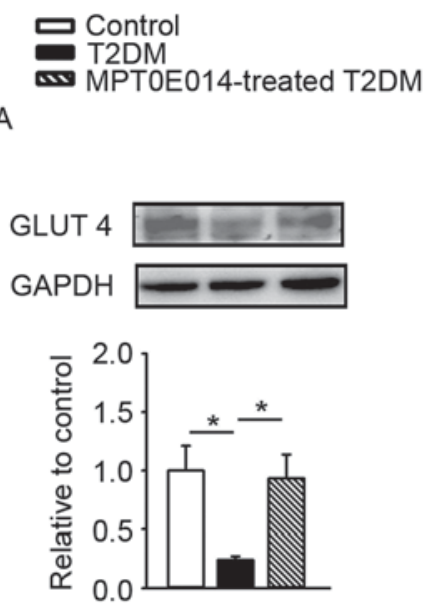

B
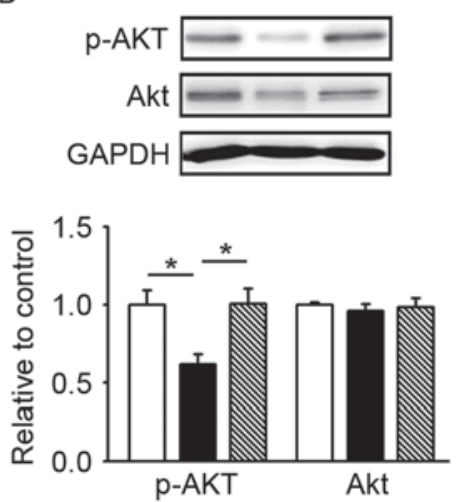

C
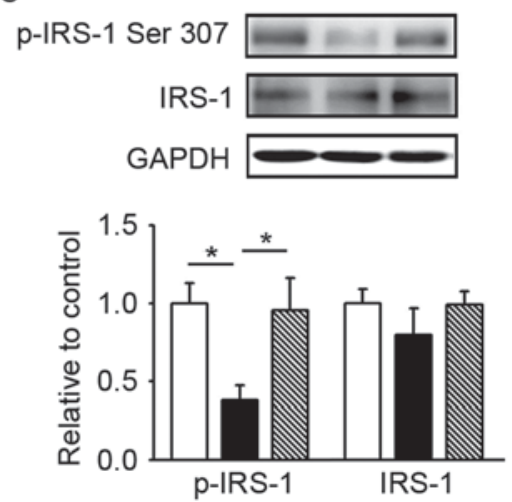

Figure 3. MPT0E014 improves insulin signaling in the T2DM heart. Cardiac (A) GLUT 4, (B) p-Akt and (C) p-IRS-1 protein levels were significantly lower in high-fat diet low-dose streptozotocin induced T2DM rats compared with the control and T2DM rats treated with MPT0E014. Data were normalized to GAPDH and are presented as the mean \pm standard error of the mean. ${ }^{*} \mathrm{P}<0.05$. $\mathrm{n}=5$ rats/group. GLUT 4, glucose transporter 4; T2DM, type 2 diabetes mellitus; p-, phosphorylated; Akt, protein kinase B; IRS-1, insulin resistant substrate.

compared with HFD + STZ-induced T2DM hearts $(\mathrm{P}<0.001$; Fig. 1B).

The role of Beclin-1 in DM cardiomyocytes was examined and it was demonstrated that the level of Beclin-1 protein was significantly lower in the hearts of HFD + STZ-induced T2DM hearts compared with the control or T2DM hearts treated with MPT0E014 ( $<<0.05$; Fig. 1C). In addition, the level of cleaved PARP1 in the T2DM cardiomyocytes was demonstrated to be increased by 0.6 compared with the control hearts; however, in the DM hearts treated with MPT0E014 the cleaved PARP protein was significantly decreased compared with the untreated DM hearts. $(\mathrm{P}<0.05$; Fig. 1D).

The regulatory role of AMPK in autophagy of the heart was examined and it was demonstrated that the expression of AMPK $\alpha 2$ was similar in the three groups. However, the p-AMPK $\alpha 2$ significantly decreased in the hearts of
HFD + STZ-induced T2DM hearts compared with the control hearts by 0.28 , and this was ameliorated in the hearts of T2DM rats treated with MPT0E014 (P<0.001; Fig. 2A). The effects of MPT0E014 on the tuberin (TSC)-mTOR signaling pathway were investigated. T2DM hearts exhibited activation of the TSC-mTOR signaling pathway, as reflected by increased protein expression of p-mTOR-S2448 (Fig. 2B) and its downstream effector p-P70S6K-Thr389 (Fig. 2C), by 0.8 and 2.5, respectively, compared with the control hearts; this effect was reversed in the MPT0E014-treated HFD + STZ T2DM hearts.

Effects of MPTOEO14 on the myocardial insulin signaling pathway, RAGE and proinflammatory cytokines. As demonstrated in Fig. 3A, the protein expression of GLUT 4 was decreased in the HFD + STZ-induced T2DM hearts compared with control hearts by 0.76 . Similar to the control hearts, hearts 
A
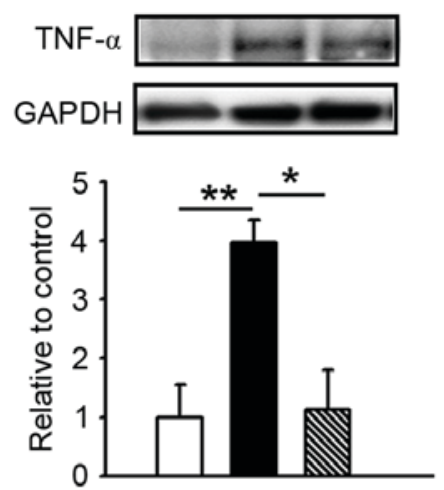

B

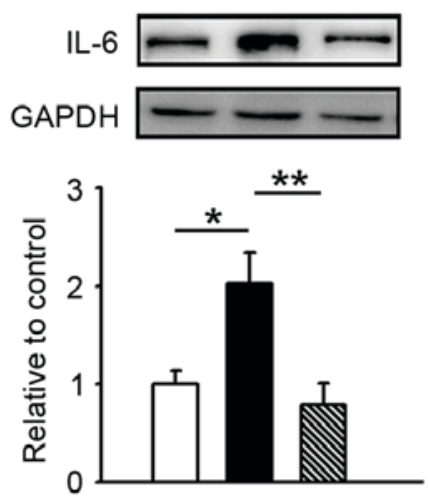

C
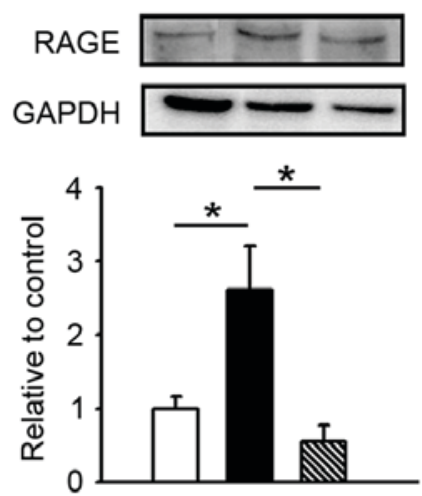

Figure 4. MPT0E014 attenuates proinflammatory cytokines and RAGE in the T2DM heart. Cardiac (A) TNF- $\alpha$, (B) IL-6 and (C) RAGE protein levels were significantly increased in the high-fat diet low-dose streptozotocin induced T2DM rats compared with the control and MPT0E014-treated T2DM rats. Data were normalized to GAPDH and are presented as the mean \pm standard error of the mean. ${ }^{*} \mathrm{P}<0.05,{ }^{* *} \mathrm{P}<0.01$. $\mathrm{n}=5$ rats/group. TNF, tumor necrosis factor; IL, interleukin; RAGE, glycosylation end product-specific receptor; T2DM, type 2 diabetes mellitus.

treated with MPT0E014 T2DM had a higher level of GLUT 4 compared with the HFD + STZ-induced T2DM hearts. In addition, the hearts of the HFD + STZ-induced T2DM rats had decreased protein expression of p-Akt (Fig. 3B) and p-IRS-1 at Ser307 (Fig. 3C), compared with the control and MPT0E014-treated T2DM hearts.

As demonstrated in Fig. 4, the hearts of the HFD + STZinduced T2DM exhibited greater protein expression of RAGE, TNF- $\alpha$, and IL- 6 compared with the control and MPT0E014-treated T2DM hearts, while the control and MPT0E014-treated T2DM hearts had similar expression of RAGE, TNF- $\alpha$, and IL-6.

\section{Discussion}

In the present study, it was demonstrated that HDAC inhibition may restore myocardial autophagy and improve insulin resistance in a T2DM rat model. T2DM is a progressive disorder that is associated with insulin resistance and is correlated with elevated, normal or low insulin levels depending on the stage when pancreatic function is measured (23). The association between T2DM and derangement in lipid metabolism, including elevated levels of triglycerides and small low-density lipoprotein-cholesterol with reduced HDL-C levels, have been reported (24,25). An increase in plasma free fatty acids is common in patients with T2DM (26) and this may contribute to insulin resistance (27). Similar to previous studies $(19,28)$, the rats in the present study fed with a HFD in a low-dose STZ T2DM model exhibited hyperglycemia with increased levels of triglycerides and free fatty acids, and decreased HDL-C levels mimicking T2DM patients, except for a nonsignificant elevation of cholesterol. In addition, a modest reduction in blood sugar and reversed alterations in plasma triglyceride and HDL-C were demonstrated following treatment with MPT0E104, suggesting antihyperglycemic and hypolipidemic action.

Autophagy is a beneficial mechanism for preserving homeostasis, and the growth and development of cells $(29,30)$.
Previous studies have demonstrated that cardiac autophagy is suppressed during metabolic derangement $(31,32)$. Beclin-1 and sequesteosome-1 (p62) are involved in the formation of autophagosomes (29) and a reduction in LC3-II levels with p62 accumulation in rodents with HFD-induced obesity was identified to indicate a decrease in autophagosome formation $(33,34)$. Similarly, the present study displayed a reduction in cardiac autophagy in T2DM rats, as indicated by decreased protein expression of LC3-II and Beclin-1. The reduction in cardiac autophagy in the present study was accompanied by suppressed myocardial phosphorylation of AMPK $\alpha 2$ and exacerbated cardiac dysfunction. The inhibition of cardiac autophagy may have been caused by AMPK dysregulation, which is involved in the pathogenesis of DM cardiomyopathy. AMPK negatively regulates mTOR activity (35) and serves an important role in mediating starvation-induced autophagy (36). Likewise, it was demonstrated that hyperglycemia increased the phosphorylation of mTOR, and activated protein expression of the mTOR downstream effector P70S6K, suggesting activation of the TSC-mTOR signaling pathway. In addition, PARP1 cleavage, a hallmark of apoptosis, was increased in the T2DM rat heart. In the present study, administration of the HDAC inhibitor MPT0E014 activated AMPKa2, and enhanced cardiac autophagic activity by modulating Beclin-1, LC3-II, TSC-mTOR and decreasing cleaved PARP1 expression. The results of the present study suggested that the restoration of autophagy following HDAC inhibition may be a novel therapeutic mechanism of MPT0E014 against DM cardiomyopathy.

RAGE accelerates vascular inflammation and cellular stress to cause atherosclerosis (37). In the present study, upregulation of the myocardial protein levels of RAGE was demonstrated, in addition to TNF- $\alpha$ and IL- 6 in T2DM rats. Additionally, it was demonstrated that treatment with MPT0E104 attenuated the upregulation of RAGE and pro-inflammatory cytokines in T2DM hearts. To the best of our knowledge, this is the first study to demonstrate the effect of HDAC inhibition on the expression of cardiac RAGE in T2DM hearts. 
Considering the important role of insulin resistance in the development of T2DM in rats fed an HFD with low-dose STZ, a reduction in the GLUT 4 protein has been implicated during insulin resistance and impaired glucose metabolism (38). Although the results of the present study demonstrated that MPT0E104 did not induce an increase in plasma insulin concentrations, the total GLUT 4 protein expression was significantly increased compared with the untreated hearts. This demonstrated that MPT0E104 was able to restore the expression of GLUT 4 protein through an insulin-independent pathway. Elevated phosphorylation of Akt and IRS-1 has been reported to be essential for the membrane translocation of GLUT $4(39,40)$. In the present study, MPT0E104-treated T2DM hearts were observed to exhibit increased GLUT 4 protein in cardiomyocytes and restoration of p-Akt and p-IRS-1 (Ser 307) protein levels. The results of the present study suggested that enhanced insulin signaling transduction may be responsible for improving insulin sensitivity by HDAC inhibition. Although MPT0E014 was able to alter the expression of cardiac GLUT 4 and the insulin signaling pathway, it may be useful to investigate the direct effects of MPT0E014 by measuring the utilization of carbohydrates.

In conclusion, HDAC inhibition improved myocardial insulin sensitivity and attenuated diabetes-induced dysregulation of cardiac autophagy. HDAC inhibition provides a novel scenario in which autophagy reactivation may represent a potential therapeutic target to reduce cardiac dysfunction in patients with DM cardiomyopathy.

\section{Acknowledgements}

The present study was supported by grants from Taipei Medical University, Wan Fang Hospital (grant nos. 104CGH-TMU-03, 104-wf-eva-03, 104swf07, 103TMU-SHH-23, 104swf02, 104-wf-eva-01 and 105-wf-eva-06) and the Ministry of Science and Technology of Taiwan (grant nos. MOST1032314-B-038-041-MY2, MOST103-2314-B-281-005-MY2, MOST103-2314-B-281-006, MOST103-2314-B-038-055, MOST104-2314-B-038-071-MY3, MOST104-2314-B-038-073, MOST104-2314-B-038-032 and MOST 105-2314-B-038-026).

\section{References}

1. Simonson DC: Etiology and prevalence of hypertension in diabetic patients. Diabetes Care 11: 821-827, 1988

2. Garcia MJ, McNamara PM, Gordon T and Kannel WB: Morbidity and mortality in diabetics in the Framingham population. Sixteen year follow-up study. Diabetes 23: 105-111, 1974.

3. Haffner SM, Lehto S, Rönnemaa T, Pyörälä K and Laakso M: Mortality from coronary heart disease in subjects with type 2 diabetes and in nondiabetic subjects with and without prior myocardial infarction. N Engl J Med 339: 229-234, 1998.

4. Yang Z and Klionsky DJ: Eaten alive: A history of macroautophagy. Nat Cell Biol 12: 814-822, 2010.

5. Maiuri MC, Zalckvar E, Kimchi A and Kroemer G: Self-eating and self-killing: Crosstalk between autophagy and apoptosis. Nat Rev Mol Cell Biol 8: 741-752, 2007.

6. Nishida K, Kyoi S, Yamaguchi O, Sadoshima J and Otsu K: The role of autophagy in the heart. Cell Death Differ 16: 31-38, 2009.

7. Gustafsson AB and Gottlieb RA: Autophagy in ischemic heart disease. Circ Res 104: 150-158, 2009.

8. Munasinghe PE, Riu F, Dixit P, Edamatsu M, Saxena P, Hamer NS, Galvin IF, Bunton RW, Lequeux S, Jones G, et al: Type-2 diabetes increases autophagy in the human heart through promotion of Beclin-1 mediated pathway. Int J Cardiol 202: 13-20, 2016.
9. Li ZL, Woollard JR, Ebrahimi B, Crane JA, Jordan KL, Lerman A, Wang SM and Lerman LO: Transition from obesity to metabolic syndrome is associated with altered myocardial autophagy and apoptosis. Arterioscler Thromb Vasc Biol 32: 1132-1141, 2012.

10. Mellor KM, Bell JR, Young MJ, Ritchie RH and Delbridge LM: Myocardial autophagy activation and suppressed survival signaling is associated with insulin resistance in fructose-fed mice. J Mol Cell Cardiol 50: 1035-1043, 2011.

11. Sciarretta S, Volpe M and Sadoshima J: Mammalian target of rapamycin signaling in cardiac physiology and disease. Circ Res 114: 549-564, 2014.

12. Kubli DA and Gustafsson AB: Cardiomyocyte health: Adapting to metabolic changes through autophagy. Trends Endocrinol Metab 25: 156-164, 2014

13. Christensen DP, Dahllöf M, Lundh M, Rasmussen DN, Nielsen MD, Billestrup N, Grunnet LG and Mandrup-Poulsen T: Histone deacetylase (HDAC) inhibition as a novel treatment for diabetes mellitus. Mol Med 17: 378-390, 2011.

14. Sharma S and Taliyan R: Histone deacetylase inhibitors: Future therapeutics for insulin resistance and type 2 diabetes. Pharmacol Res 113: 320-326, 2016.

15. Kao YH, Liou JP, Chung CC, Lien GS, Kuo CC, Chen SA and Chen YJ: Histone deacetylase inhibition improved cardiac functions with direct antifibrotic activity in heart failure. Int $J$ Cardiol 168: 4178-4183, 2013

16. Lee TI, Kao YH, Tsai WC, Chung CC, Chen YC and Chen YJ: HDAC inhibition modulates cardiac PPARs and fatty acid metabolism in diabetic cardiomyopathy. PPAR Res 2016: 5938740, 2016.

17. Brownlee M: Biochemistry and molecular cell biology of diabetic complications. Nature 414: 813-820, 2001.

18. Barlovic DP, Soro-Paavonen A and Jandeleit-Dahm KA: RAGE biology, atherosclerosis and diabetes. Clin Sci (Lond) 121: 43-55, 2011.

19. Mansor LS, Gonzalez ER, Cole MA, Tyler DJ, Beeson JH, Clarke K, Carr CA and Heather LC: Cardiac metabolism in a new rat model of type 2 diabetes using high-fat diet with low dose streptozotocin. Cardiovasc Diabetol 12: 136, 2013.

20. Lee TI, Kao YH, Chen YC, Pan NH, Lin YK and Chen YJ: Cardiac peroxisome-proliferator-activated receptor expression in hypertension co-existing with diabetes. Clin Sci (Lond) 121: 305-312, 2011

21. Lee TI, Chen YC, Kao YH, Hsiao FC, Lin YK and Chen YJ: Rosiglitazone induces arrhythmogenesis in diabetic hypertensive rats with calcium handling alteration. Int J Cardiol 165: 299-307, 2013.

22. Lkhagva B, Lin YK, Kao YH, Chazo TF, Chung CC, Chen SA and Chen YJ: Novel histone deacetylase inhibitor modulates cardiac peroxisome proliferator-activated receptors and inflammatory cytokines in heart failure. Pharmacology 96: 184-191, 2015.

23. Cefalu WT: Animal models of type 2 diabetes: Clinical presentation and pathophysiological relevance to the human condition. ILAR J 47: 186-198, 2006.

24. Boden G, Lebed B, Schatz M, Homko C and Lemieux S: Effects of acute changes of plasma free fatty acids on intramyocellular fat content and insulin resistance in healthy subjects. Diabetes 50: 1612-1617, 2001.

25. Haffner SM; American Diabetes Association: Management of dyslipidemia in adults with diabetes. Diabetes care 26 (Suppl 1): S83-S86, 2003

26. Reaven GM, Hollenbeck C, Jeng CY, Wu MS and Chen YD: Measurement of plasma glucose, free fatty acid, lactate, and insulin for $24 \mathrm{~h}$ in patients with NIDDM. Diabetes 37: 1020-1024, 1988.

27. Shulman GI: Cellular mechanisms of insulin resistance. J Clin Invest 106: 171-176, 2000

28. Srinivasan K, Viswanad B, Asrat L, Kaul CL and Ramarao P. Combination of high-fat diet-fed and low-dose streptozotocin-treated rat: A model for type 2 diabetes and pharmacological screening. Pharmacol Res 52: 313-320, 2005.

29. Levine B and Kroemer G: Autophagy in the pathogenesis of disease. Cell 132: 27-42, 2008

30. Singh R and Cuervo AM: Autophagy in the cellular energetic balance. Cell Metab 13: 495-504, 2011.

31. Sciarretta S, Zhai P, Shao D, Maejima Y, Robbins J, Volpe M, Condorelli $G$ and Sadoshima J: Rheb is a critical regulator of autophagy during myocardial ischemia: Pathophysiological implications in obesity and metabolic syndrome. Circulation 125: 1134-1146, 2012 
32. He C, Zhu H, Li H, Zou MH and Xie Z: Dissociation of Bcl-2-Beclin1 complex by activated AMPK enhances cardiac autophagy and protects against cardiomyocyte apoptosis in diabetes. Diabetes 62: 1270-1281, 2013

33. Guo R, Zhang Y, Turdi S and Ren J: Adiponectin knockout accentuates high fat diet-induced obesity and cardiac dysfunction: Role of autophagy. Biochim Biophys Acta 1832: 1136-1148, 2013.

34. Xu X and Ren J: Macrophage migration inhibitory factor (MIF) knockout preserves cardiac homeostasis through alleviating Akt-mediated myocardial autophagy suppression in high-fat diet-induced obesity. Int J Obes (Lond) 39: 387-396, 2015.

35. Inoki K, Zhu T and Guan KL: TSC 2 mediates cellular energy response to control cell growth and survival. Cell 115: 577-590, 2003.

36. Lum JJ, DeBerardinis RJ and Thompson CB: Autophagy in metazoans: Cell survival in the land of plenty. Nat Rev Mol Cell Biol 6 : 439-448, 2005

37. Sun L, Ishida T, Yasuda T, Kojima Y, Honjo T, Yamamoto Y, Yamamoto H, Ishibashi S, Hirata K and Hayashi Y: RAGE mediates oxidized LDL-induced pro-inflammatory effects and atherosclerosis in non-diabetic LDL receptor-deficient mice. Cardiovasc Res 82: 371-381, 2009.
38. Zisman A, Peroni OD, Abel ED, Michael MD, Mauvais-Jarvis F, Lowell BB, Wojtaszewski JF, Hirshman MF, Virkamaki A, Goodyear LJ, et al: Targeted disruption of the glucose transporter 4 selectively in muscle causes insulin resistance and glucose intolerance. Nat Med 6: 924-928, 2000.

39. Lin HV, Ren H, Samuel VT, Lee HY, Lu TY, Shulman GI and Accili D: Diabetes in mice with selective impairment of insulin action in Glut4-expressing tissues. Diabetes 60: 700-709, 2011.

40. Chao KC, Chao KF, Fu YS and Liu SH: Islet-like clusters derived from mesenchymal stem cells in Wharton's Jelly of the human umbilical cord for transplantation to control type 1 diabetes. PLoS One 3: e1451, 2008. 